\title{
Short-Term Power Load Forecasting of Least
}

\section{Squares Support Vector Machine Based on Wavelet Transform and Drosophila Algorithm}

\author{
Jian-Na ZHAO \\ Department of Economics and Management, North China \\ Electric Power University \\ Baoding, 071003, China \\ zhjnzhf@163.com
}

\author{
Xiao-Bo HE * \\ Department of Economics and Management, North China \\ Electric Power University \\ Baoding, 071003, China \\ hxb8858460@163.com
}

\begin{abstract}
As an energy that can't be stored and related to the national economy and the people's livelihood, the stability of electric energy has been paid more and more attention in our country. In order to solve this problem, a short-term least squares support vector machine (SVM) based on wavelet decomposition and Drosophila algorithm is proposed to predict short-term power load. The example shows that WT-FOA-LSSVM has been improved obviously in the prediction precision, and has certain applicability.
\end{abstract}

Keywords-Power load forecasting, Wavelet transform, Fruit fly algorithm

\section{INTRODUCTION}

Power load forecasting is an important part of management modernization of electric power systems, which has attracted more and more attentions from the academic and the practice. High precision power load forecasting can solve the contradiction between relieving the balance of power supply and demand, providing a solid foundation for the stability and reliability of power grid, avoiding the waste of resources in the process of power grid dispatching, and improving the economic benefits.

With the continuous improvement of the accuracy requirement of electric load forecasting in power grid operation, intelligent algorithm has attracted the attention of scholars. Neural network prediction models, grey models and least squares support vector machines are commonly used. [1] In order to improve the accuracy of power load forecasting, this paper uses FOA algorithm to optimize LSSVM's kernel function parameters and penalty parameters for short-term power load prediction, avoiding blindness of LSSVM parameter selection and improving the prediction ability of the model.

\section{A BRIEF INTRODUCTION OF WT-FOA-LSSVM MODEL}

A. WT

Wavelet Transform(WT), it has been developed in recent decades. It is a kind of mathematical analysis method of signal. It can analyze the signal localization in time and frequency domain simultaneously, and it has the characteristic of multiresolution analysis. The wavelet transform can easily realize the decomposition of the signal, the signal is divided into approximations and details, the approximate part refers to the signal can be projected onto a large scale (i.e. larger scale a) low frequency components in space; the details refer to the high frequency component of the signal corresponding to the small scale space. [2] As shown in Fig.1:

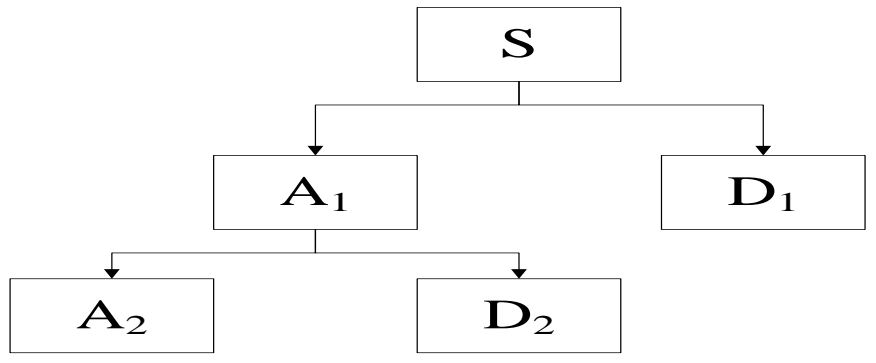

Fig. 1. Wavelet decomposition

\section{B. $L S-S V M$}

Least squares support vector machine(LS-SVM). It uses the sum of squares of the least squares linear system error as the loss function, and solves the solution process to understand a set of equational equations, which speeds up the solving speed and solves less computing resources. [3] From training LS-SVM problems, kernel function parameters and penalty parameters are generally set according to experience, which leads to randomness and inaccuracy in the application of LS-SVM algorithm. [4] In order to solve this problem, this paper uses the fruit fly algorithm to optimize these two parameters, making the prediction accuracy of LS-SVM improved.

C. FOA

The basic idea of FOA is that fruit flies perceive the surrounding food concentration according to their location. By comparing the taste concentration, Drosophila flies near the location of maximum or minimum concentration, and iterate iteratively through food concentration, so as to get the extreme value of the objective function. [5] The process of finding food for the Drosophila population is shown as shown in the diagram: 


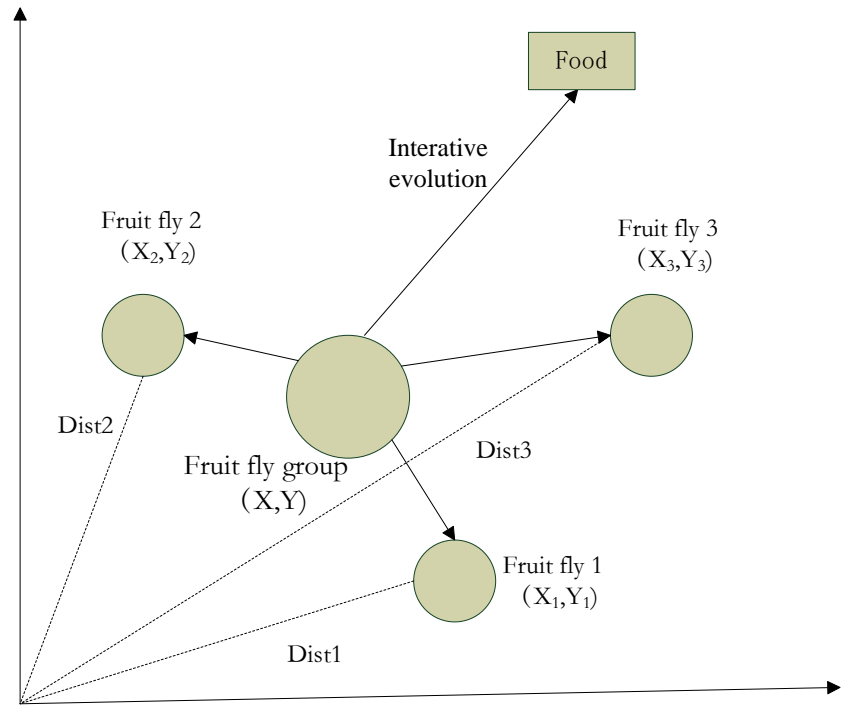

Fig. 2. Food finding iterative process of a fruit fly swarm

\section{INTRODUCTION OF WT-FOA-LSSVM MODEL BUILDING}

Fig. 3 is a flowchart of the WT-FOA-LSSVM model, and its specific steps are as follows:

1) WT algorithm handles load raw data

2) Algorithm parameters for the initialization of Drosophila:

The Drosophila algorithm mainly includes the initial position of the Drosophila population, the Drosophila searching for the random direction and distance of the food, the size of the population, and the maximum number of iterations.

3) Optimization of fruit fly algorithm LSSVM:

The two parameters that need to be optimized in LSSVM are: penalty value $\gamma$ and nuclear parameters $\sigma^{2}, \gamma=20 * S(i .1)$, $\sigma^{2}=\mathrm{S}(\mathrm{i}, 2)$, bring into the training LSSVM. Set up the fitness function and use RMSE as the fitness function value, as shown by formula 1 :

$$
\text { RMSE }=\sqrt{\frac{\sum_{i=1}^{n}\left(y_{i}-\hat{y}_{i}\right)^{2}}{n}}
$$

The actual value of $y_{i}$ as a point $\mathrm{i}$ point load, Load forecast for $\hat{y}_{i}$ as a point $\mathrm{i}, \mathrm{n}$ is the number of predicted data,
If the maximum number of iterations or $\mathrm{RMSE}<0.01 \%$ is reached, the termination calculation is optimal and the optimal sum is obtained.

\section{4) LSSVM model prediction:}

The optimized parameters $\gamma, \sigma^{2}$, and test data were brought into the LSSVM for prediction, and the results were finally obtained.

\section{CASE STUDY}

This paper selects 24 hours load data from May 1, 2014 to July 23, 2014 in Hengshui, Hebei Province, with 1986 load data from May 1st to July 22nd as sample data, and July 23rd load as model validation data. Because people's production and life activities will be interfered by external factors such as weather, temperature, holidays and so on, which will affect the electric load. Therefore, we consider the local temperature, weather conditions and the three factors of holidays. [6]

\section{A. Parameters Setting}

In order to show the superiority of wt-foa-lssvm prediction model, this paper introduces four prediction models of foa-lssvm, PSO-LSSVM, LSSVM and BPNN for comparison and analysis, and draws the advantages and disadvantages of each algorithm through comparison. The parameters of the above algorithm are set as shown in the Table I.

\section{B. Model performance evaluation}

In order to test the validity of the model, this paper will examine the accuracy of the prediction results from two indexes, mean absolute percentage error(MAPE) and mean square error(MSE):

$$
\begin{aligned}
& M A P E=\frac{1}{n} \sum_{i=1}^{n}\left|\frac{\hat{y}_{i}-y_{i}}{y_{i}}\right| \\
& M S E=\frac{1}{n} \sum_{i=1}^{n}\left(\hat{y}_{i}-y_{i}\right)^{2}
\end{aligned}
$$

Where yi represents the actual value at period $\mathrm{i}$; $\mathrm{y} \hat{\mathrm{i}}$ is the forecasting value at period $i$ and $n$ is the number of forecasting period. Forecasting results are shown table II. Fig. 3 and Fig. 4 are the contrasts between the WT-FOA-LSSVM prediction model and the other four models. 


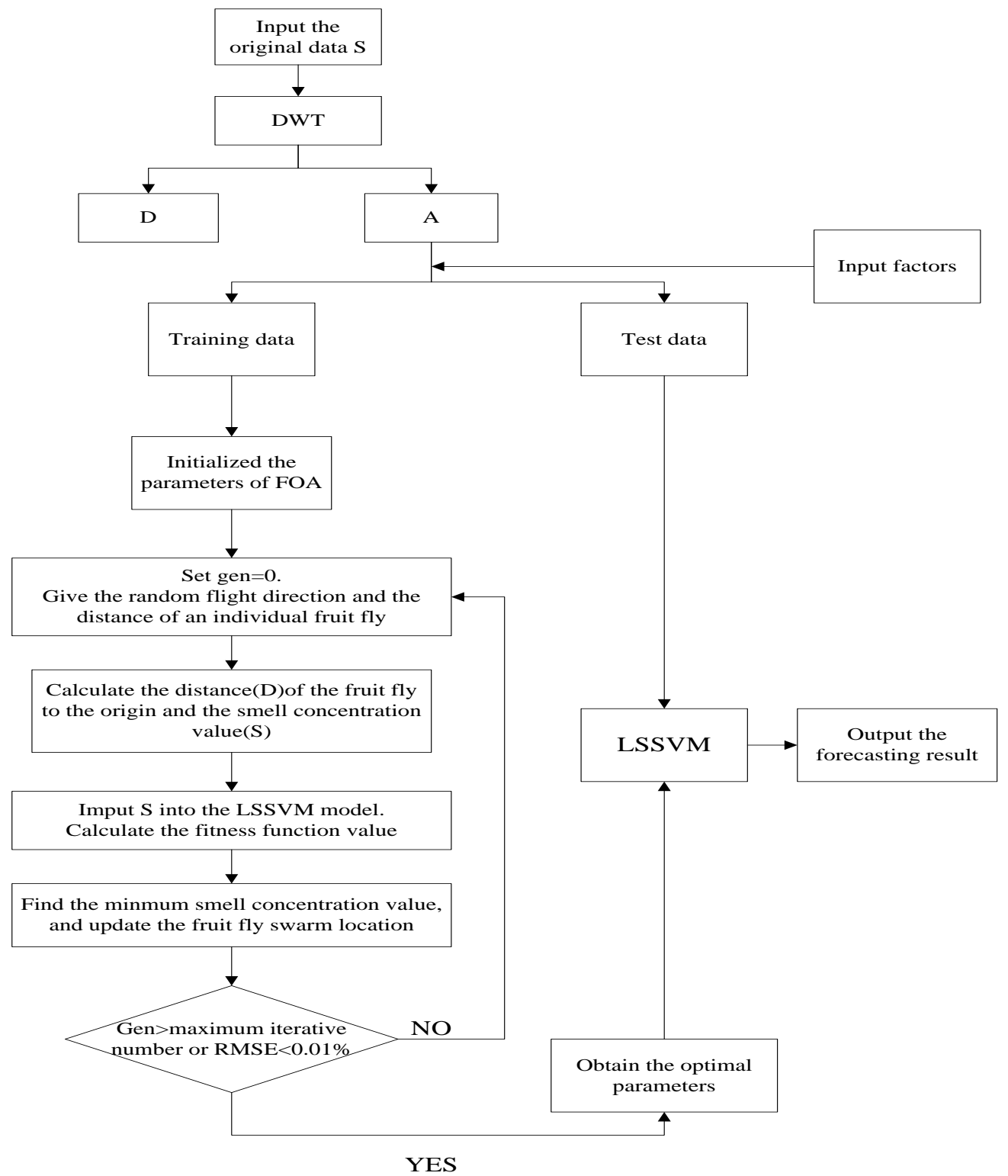

Fig. 3. Flowchart of the WT-FOA-LSSVM modeling

TABLE I. PARAMETERS OF MODELS MENTIONED IN THE PAPER

\begin{tabular}{|c|c|}
\hline Model & Parameters \\
\hline FOA-LSSVM & $\begin{array}{c}\text { Initial-location[rands(1,2),rands(1,2)];FR=[-10.1 } \\
0] ; \text { sizepop=50; } N_{\max }=100\end{array}$ \\
\hline PSO-LSSVM & $\begin{array}{c}C_{1}=C_{2}=2 ; N_{\max }=200 ; \\
\text { sizepop }=20 ; w_{0}=0.8 ; w_{n}=0.3\end{array}$ \\
\hline LSSVM & $\mathrm{C}=40 ; \sigma^{2}=5$ \\
\hline BPNN & $\begin{array}{l}\text { Nmax=100;hidden layer node=[5,5];learning } \\
\text { rate=0.1;goal=0.00004 }\end{array}$ \\
\hline
\end{tabular}

TABLE II. MODELS PERFORMANCE EVALUATIONS

\begin{tabular}{|c|c|c|}
\hline Model index & MAPE & MSE \\
\hline WT-FOA-LSSVM & $1.068 \%$ & 130.4913 \\
\hline WT-LSSVM & $1.111 \%$ & 147.9492 \\
\hline FOA-LSSVM & $1.3534 \%$ & 181.4398 \\
\hline PSO-LSSVM & $1.414 \%$ & 191.3687 \\
\hline LSSVM & $1.8457 \%$ & 300.9418 \\
\hline BPNN & $1.674 \%$ & 268.3937 \\
\hline
\end{tabular}




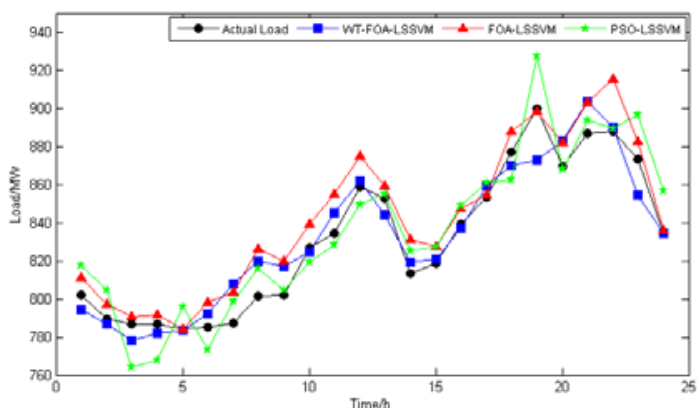

Fig. 4. Actual load and forecasting results of WT-FOA-LSSVM, FOA-LSSVM and PSO-LSSVM

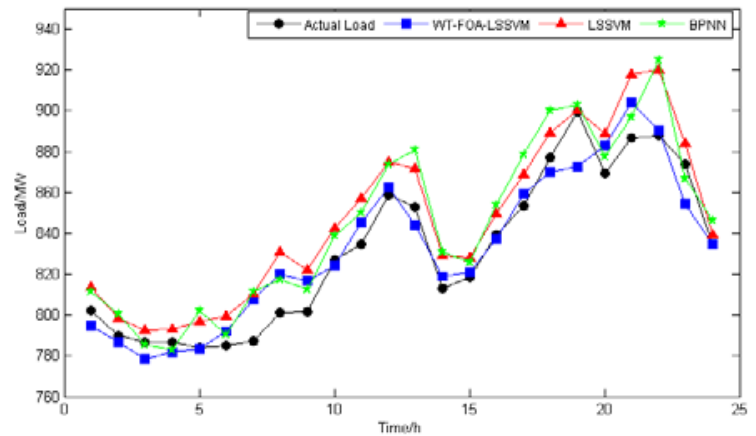

Fig. 5. Actual load and forecasting results of WT-FOA-LSSVM, LSSVM and BPNN

From Table IV, we can see that the MAPE of the WT-FOA-LSSVM prediction model is $1.068 \%$, which is lower than the four models of FOA-LSSVM, PSO-LSSVM, LSSVM and BPNN, the MSE of which is 130.4913, which is also lower than the other four models. It can be seen that the stability of the WT-FOA-LSSVM prediction model and the prediction accuracy are superior to those of the other four models, and can be used for short-term power load forecasting.

\section{V.CONCLUSION}

In order to strengthen the stability and economic type of the power grid and avoid the waste in the scheduling process, it is of profound significance to improve the precision of power load forecasting. Because the short-term power load is disturbed by many external factors, it has the characteristics of irregular and fluctuating. Therefore, single model can't be applied to predict the electric load. [7] According to the power load and the least squares parameter selection characteristics of blindness, this paper proposes a wavelet transform and least squares support vector machine algorithm Drosophila to carry out prediction of short-term power load based on the prediction results show that the relative error was [-3\%, 3\%], MAPE, MSE were better than those of the other four kinds of model has higher prediction accuracy. To sum up, the wt-foa-lssvm prediction model has the characteristics of high prediction accuracy and simple operation, which provides a scientific and effective reference for short-term power load forecasting.

\section{REFERENCES}

[1] Genetic optimization of neural network prediction method and its application _ Niu Dongxiao

[2] Research on combination forecasting model of BP neural network based on genetic algorithm optimization_Liang yi

[3] Adaptive hybrid genetic optimization BP algorithm for short term power load forecasting_Shi yingling

[4] Optimization model design of Daqing rainfall neural network based on genetic algorithm and BP algorithm_Li hongwei.

[5] Application of genetic neural network in short term load forecasting of electric power system_Liang haifeng

[6] Short term load forecasting of power system based on genetic optimization neural network_Wang wu

[7] Long term electric load forecasting and analysis based on genetic algorithm and BP neural network in urban area_Cheng yugui 